\title{
The Influence of Subjects' Personality Traits on Personal Spatial Zones in a Human-Robot Interaction Experiment *
}

Michael L Walters, Kerstin Dautenhahn, René te Boekhorst, Kheng Lee Koay, Christina Kaouri, Sarah Woods,

Chrystopher Nehaniv, David Lee, Iain Werry

Adaptive Systems Research Group

University of Hertfordshire

Hatfield, Herts. UK

\{M.L.Walters, K.Dautenhahn, R.TeBoekhorst\}@herts.ac.uk

\begin{abstract}
In the present study we investigated humanrobot and robot-human approach distances. We found that subjects' personality profiles influence personal spatial zones in human-robot interaction experiments. We tested two hypotheses: First, we predicted that approach distances preferred by humans when interacting with a robot would be comparable to those preferred when humans interact socially with each other. Our experiments involving humans interacting with a mobile robot confirm this hypothesis. However, surprisingly, a large minority of subjects in the experiments took up positions which were significantly closer, suggesting that they were not treating the robot as a 'social entity'. We then tested the hypothesis that common personality factors exist which could be used to predict subjects' likely approach distance preferences. The subjects' personalities were assessed using several traits from the threefactor Eysenck personality model. Further analysis of the data identified four new factors, different from Eysenck's model, tentatively labeled "Proactiveness", "Social Reluctance", "Timidity" and "Nervousness". When testing for correlations between approach distances and personality data, "Proactiveness" correlates with social distance, i.e. subjects that score higher on this factor come less close to the robot. We discuss the potential suitability of personality factors to predict approach distances in human-robot interaction experiments.
\end{abstract}

Index Terms - Human-Robot Interaction, Social Robot, Social Spaces, Personal Spaces

\section{INTRODUCTION}

Studying social and personal spaces with regard to robots, designed for use in the home, is a particular area of research within the wider field of Human - Robot Interaction (HRI). In the near future, it is anticipated that robots will increasingly be used for applications in office and domestic environments. Therefore they will be required to work alongside and interact closely with the human residents [1]. As the study of socially interactive robots is relatively new, there is not a large body of established theories, methods and research experience to draw upon, so experimenters in the field usually use existing research into human-human social interactions as a starting point. These methods and results, along with later research, have provided a guide for more recent research, studies and investigations into human reactions to and attitudes towards robots [2] - [16].

\section{A. Human-Robot Social Spaces}

The main emphasis of our research is on the physical, spatial, visual and audible non-verbal social aspects of robots interacting socially with humans. In particular, we are interested in studying human-robot social spaces and distances. Hall [17] described a basis for research into social and personal spaces between humans, and later work in psychology has demonstrated that social spaces substantially reflect and influence social relationships and attitudes of people. Embodied non-verbal interactions, such as approach, touch, and avoidance behaviours, are fundamental to regulating human-human social interactions [18]. Spatial zones among people are strongly influenced by cultural factors. The generally recognized personal spatial zones between humans are well known and are summarized (for northern Europeans) in Table 1 from Lambert [19].

TABLE 1

Human-Human Personal Spatial Zones

\begin{tabular}{|l|l|l|}
\hline Personal Spatial Zone & Range & Situation \\
\hline Close Intimate & 0 to $0.15 \mathrm{~m}$ & Lover or close friend touching \\
\hline Intimate Zone & $0.15 \mathrm{~m}$ to $0.45 \mathrm{~m}$ & Lover or close friend only \\
\hline Personal Zone & $0.45 \mathrm{~m}$ to $1.2 \mathrm{~m}$ & Conversation between friends \\
\hline Social Zone & $1.2 \mathrm{~m}$ to $3.6 \mathrm{~m}$ & Conversation to non-friends \\
\hline Public Zone & $3.6 \mathrm{~m}+$ & Public speech making \\
\hline
\end{tabular}

This paper presents our exploratory research into humanrobot social spaces, investigating whether human-human personal spatial zones transfer to human-robot interaction. As a starting point we have compared human-robot approach distances to those that would be expected for the case of a human approaching another human. A working hypothesis that human-robot interpersonal distances would be comparable to those found for human-human interpersonal distances was used; cf. Walters et al. [19], Christensen and Pacchierotti [21]. We expected that in scenarios designed for direct human-robot interaction, people would assume distances that on average correspond to the 'Social' or 'Personal' zone (similar to the distances people use when having face-to-face conversations with each other) thus treating the robot as a 'social being' with respect to social distances.

\section{HumAN-Robot SOCIAL DistanCE EXPERIMENTS}

\footnotetext{
* The work described in this paper was conducted within the EU Integrated Project COGNIRON ("The Cognitive Robot Companion") and was funded by the European Commission Division FP6-IST Future and Emerging Technologies under Contract FP6-002020.
} 
The human-robot social space experiments were performed prior to a separate series of experimental sessions studying human-robot interactions in a range of task based scenarios. The subject sample set consisted of 28 adult volunteers (staff members and students from University of Hertfordshire). The sample was balanced for age, gender and whether subjects had a technology related background. All subjects completed consent forms and were not paid for participation.

\section{A. The Robot:}

The robot used for this study was a commercially available PeopleBotTM robot which is mechanistic in appearance (see Fig. 1). This is a human scaled robot, $1.1 \mathrm{~m}$ tall, with a camera with pan and tilt capabilities. The robot was also fitted with three banks of eight sonar range finders which allow the robot to sense objects at low level (approximately $0.25 \mathrm{~m}$ from the ground) all around, and at high level (at a height of approximately $1 \mathrm{~m}$ ) in front. The sonar sensors are particularly good at sensing soft targets, such as humans and semi hard materials such as walls, furniture etc, and are primarily used for object avoidance and safe movement in environments containing humans. The robot is steered by two differential driving wheels, and has a caster wheel at the back and front to provide stability. The only anthropomorphic feature of the robot was a lifting arm, with a hook type endeffector, to allow the robot to fetch and carry small objects in specially adapted pallets. The robot was operated under remote control by two hidden operators. This is commonly called Wizard of Oz (WoZ) and is a technique that is widely used in HRI studies. It provides a very flexible way to implement complex robot behaviour within a quick time-scale [22][23]. The main advantage is that it saves considerable time over programming a robot to carry out complex interactions fully autonomously.

At the start of each experiment the robot was driven to the same fixed position in the room for each approach distance test. This was achieved by using the table in the corner of the room (position 5 in Figure 3) as a stop position reference for the robot's sonar range sensors. The robot could then therefore be driven towards the corner, until it stopped at a fixed distance from the corner.

\section{B. Experimental Method:}

The experimental sessions took place in a conference room at the University premises, which was converted and furnished to resemble a domestic sitting room as far as was possible. One end of the room was partitioned off using shelf units, cupboards and high screens to form a control area for the robot operators. Marks were made on the floor using masking tape along the diagonal of the experiment room, and scale marks made at $0.5 \mathrm{~m}$ intervals between them (Figs. 2 and $3)$. The experiments were supervised by an experimenter who introduced and explained the tests to be carried out to the subject. Otherwise, she interfered as little as possible with the actual experiment.

The human-robot comfort and approach distances were estimated from video records of the sessions, rather than having the experimenter making intrusive measurements or notes during the experimental sessions.

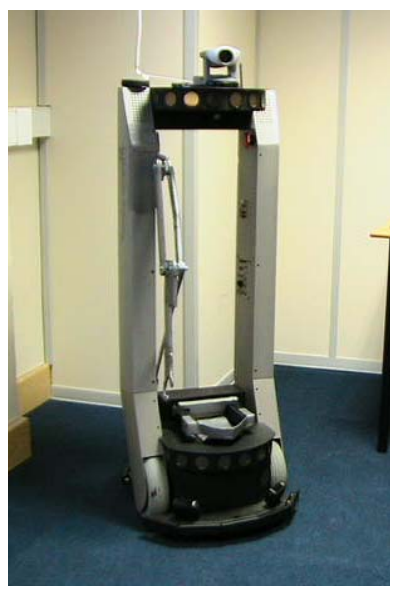

Fig 1. The PeopleBotTM robot used in the experiments.

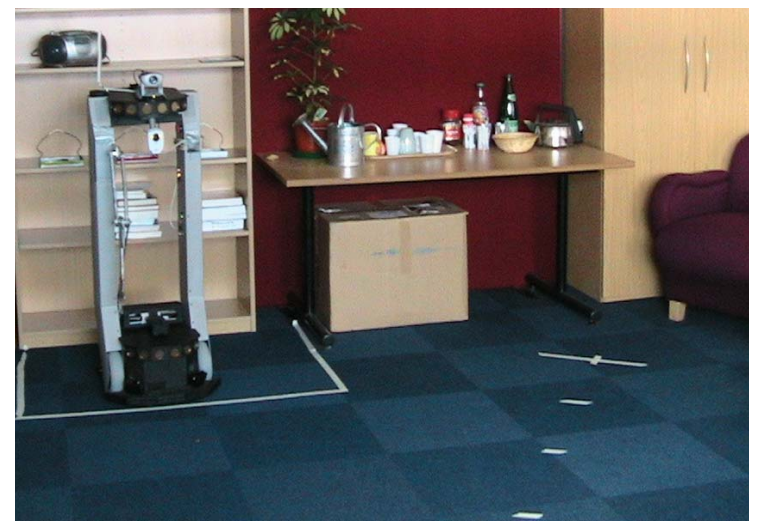

Fig 2. View of the simulated living room showing robot and the $0.5 \mathrm{~m}$ scale marked diagonally on the floor

Each experimental session followed the same format: 1) Entry to room and introduction of robot, 2) Co-habituation and initial questionnaires; While the subject was filling in the first questionnaires, the robot wandered randomly around the test area to acclimatise the subject to the robot, for a period of five to ten minutes prior to the distance tests, 3) Comfort and social distance tests, 4) Various other HRI task scenarios and questionnaires ${ }^{1}$.

For measuring the human subject's comfort threshold distance when approaching the robot, the robot was driven to point 5 (Figure 3), next to the corner table and turned to face along the distance scale towards point 4 (Figure 3). The subject was told to start at point 4 and to move towards the robot until he or she felt that they were at a comfortable distance away from the robot (Figure 4). The instruction used was "Move towards the robot as far as you feel comfortable to do so". Next, they were told to move as close to the robot as they physically could (if not already in that position); "Move as close to the robot as you physically can". Then they were told to move away again to a comfortable distance; "move back to your most comfortable distance". They were then told

\footnotetext{
${ }^{1}$ These latter parts were carried out for separate HRI investigations and are therefore not considered in this paper.
} 
to repeat these steps once again as a consistency check. The comfortable approach, closest physical and comfortable withdrawal distances were measured for each of the two tests by later close observation of the video records. The distances were estimated to the nearest $0.25 \mathrm{~m}$ (accuracy $\pm 0.125 \mathrm{~m}$ ).

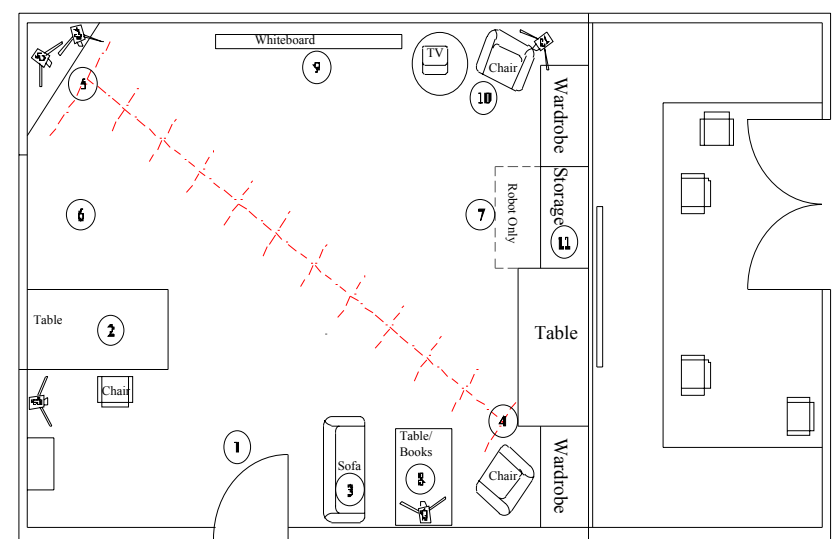

Fig 3. Plan view of simulated living room layout. Comfort distance tests carried were out along the marked diagonal line.

For the human-robot approach distance experiments two measurements for the comfortable approach distance and two for the comfortable withdrawal distance were obtained. In practically all cases subject's withdrawal distances were within $0.25 \mathrm{~m}$ of their comfortable approach distances. The four approach and withdrawal distances were then aggregated to produce a single mean comfortable approach distance value for each subject.

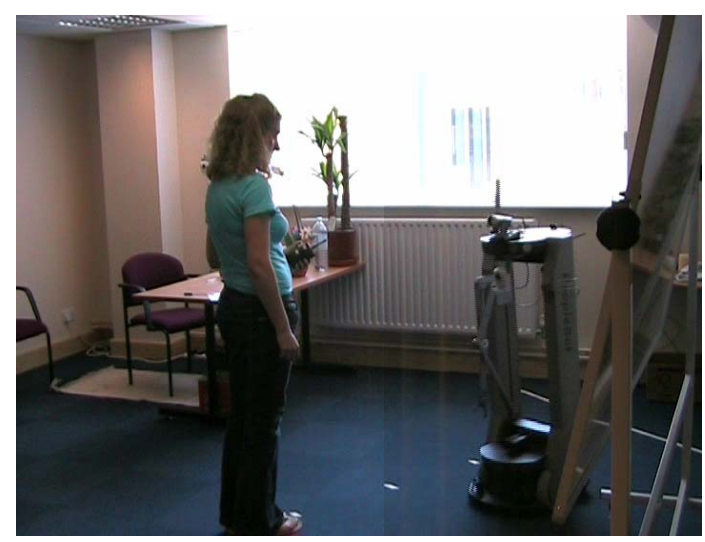

Fig 4. Human-robot approach distance experiment; a human subject approaching the robot

A second set of comfortable approach distance measurements were then made for the situation where the robot approached a stationary human subject. The subject was asked to stand at position 4), and the robot was driven to position 5) (the diagonally opposite corner of the room. see Fig. 3). The subject was then asked to say "Stop" when the robot came as close as they felt was comfortable. The robot was then driven directly towards the subject at a speed of approximately 1 meter per second. When the subject said "Stop" the robot was stopped as quickly as the WoZ operators could react. This usually involved an overshoot of $0.5 \mathrm{~m}$ or more. The distance of the robot from the subject at the instant when the subject actually said "Stop" was estimated from the video records of the experiment. By using the video system stop frame facility and the $0.5 \mathrm{~m}$ scale marks on the floor it was possible to estimate the distance to the nearest $0.25 \mathrm{~m}$ (accuracy $\pm 0.125 \mathrm{~m}$ ). The robot-human approach distance experiment was also repeated twice as a consistency check. Two robot-human comfortable approach distance measurements were obtained, which were then aggregated to obtain a single mean distance value for each subject.

\section{Results}

The means of the four human to robot comfortable approach distance results obtained were calculated for each subject and a frequency histogram was plotted, with the ranges set at $0.25 \mathrm{~m}$ intervals (consistent with the accuracy of the measurements). The results are shown in Figures 5 and 6 . For the case of the robot approaching the human the means of the two distances for each subject are shown in Figure 7. There was no robot to human approach distance less than $0.5 \mathrm{~m}$ as the robot's anti-collision safety system prevented it moving closer than $0.5 \mathrm{~m}$ to a human (or any other object).

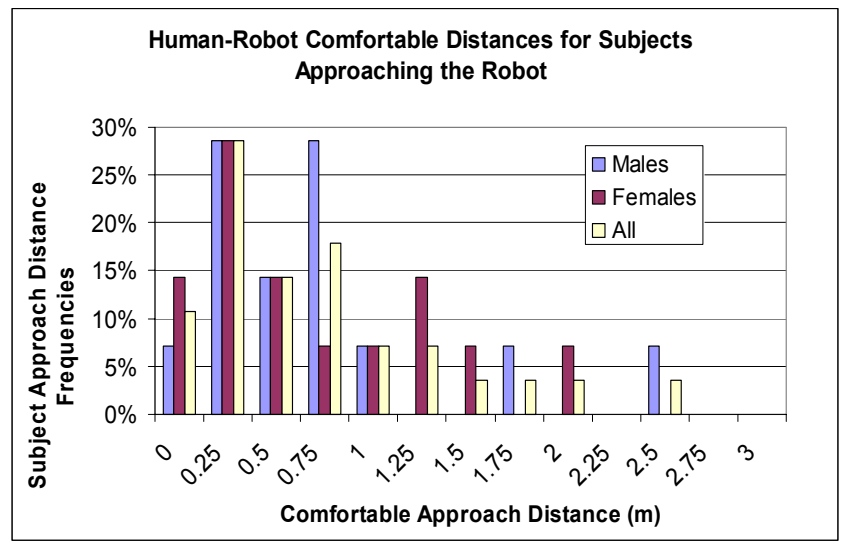

Fig 5. Comfortable distance frequencies for subjects approaching the robot. (Shown as percentages of the subject sample set: $N=28, M=14, F=14$ )

The approach distance to the robot for the majority of subjects $(60 \%$ total) was within the expected ranges for comparable human-human social distances, corresponding to either the personal or social spatial zones. However, approximately $40 \%$ of subjects approached the robot to a distance of less than $0.45 \mathrm{~m}$. Also, $38 \%$ of the subjects allowed the robot to approach right up to the $0.5 \mathrm{~m}$ limit set by the robot's safety system. The fact that they did not stop the robot from physically approaching so closely indicates that the robot did not make these subjects feel threatened or uncomfortable. Indeed, if another, unfamiliar human (a stranger) was to approach to the same close distance; most humans would start to feel distinctly uncomfortable and threatened. Practically all the subjects stated that they did not feel threatened by the robot (and only a minority wished to become intimate with the robot in the sense of having the robot as a friend or companion). It is probable therefore, that this large minority of 
subjects did not relate to the PeopleBotTM in terms of the normal social distances between humans, i.e. reflecting a conversation between friends or acquaintances.

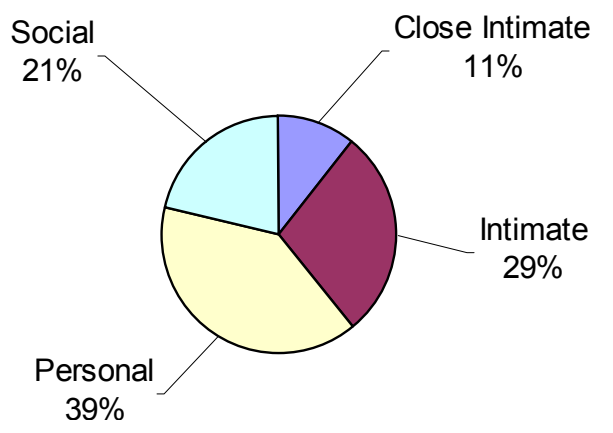

Fig 6. Human to robot comfortable approach distances categorised into Hall's personal spatial zones. (Shown as percentages of the subject sample set: $\mathrm{N}=28)$

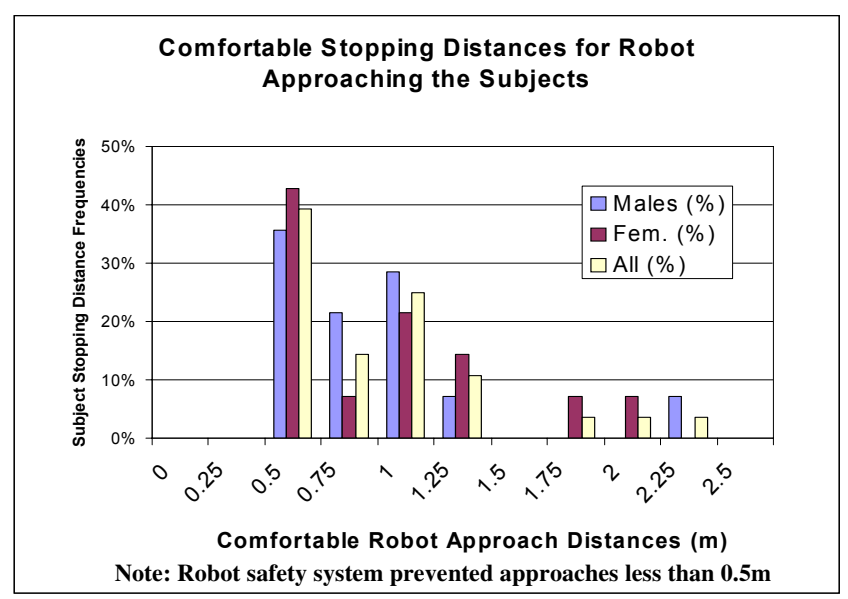

Fig 7. Social (stopping) distance frequencies when the robot approaches the human subjects. (Shown as percentages of the subject sample set: $\mathrm{N}=28$, $\mathrm{M}=14, \mathrm{~F}=14)$.

\section{Social Distance and Subjects' Personalities}

In order to address the issue of personality, we chose Eysenck's Three-Factor Psychoticism, Extroversion and Neuroticism (PEN) model as a starting point [24]. In Eysenck's view, personality types are not categories that a few people fit; rather, types are dimensions that span a space in which persons can be pinpointed at all possible positions [25]. Types tend to be normally distributed, meaning that they can take continuous values and most people fall around the average mark. From his studies with human subjects Eysenck concluded that personality can be understood in terms of three basic personality factors, which in turn are composed of a number of traits. Due to the time constraints for the HRI experiments not all 27 Eysenck traits were used. Also, since the subjects were rating themselves, certain traits were not considered suitable for self-assessment (e.g. antisocial). Moreover, we considered only traits that could be applied to rating human as well as robot personality [16]. The following traits were chosen that seemed most relevant to the present study:
Psychoticism: The loss of distortion of reality and the inability to distinguish between reality and fantasy. This is not a dimension like the other two (it does not consist of polar opposites) - it is present in all individuals to some degree. The traits associated are: Aggressive, Cold, Egocentric, Impersonal, Impulsive, Antisocial, Un-empathetic, Creative and Tough-minded. The traits selected to be used for our study were: Aggressiveness, Impulsiveness and Creativity.

Extroversion: Degree to which a person is outgoing and participative in relating to others. Traits associated comprise: Sociable, Lively, Active, Assertive, Sensation Seeking, Carefree, Dominant, Surgent and Venturesome. The traits selected to be used for our study were: Sociability, General Activity Level, Assertiveness, Excitement-Seeking and Dominance.

Neuroticism: An individual's adjustment to environment and stability of behaviour over time. The traits associated are: Anxious, Depressed, Guilt Feelings, Low Self Esteem, Tense, Irrational, Shy, Moody and Emotional. The traits selected to be used for our study were: Anxiety, Tension, Shyness and Emotional Vulnerability.

The subject personality questionnaire required the participants to rate themselves in terms of the 12 different personality traits using a 5-point Likert scale. Subjects were all informed that this information would be treated confidentially and would not be linked to their real name during any stage of the evaluation.

The score for each personality factor (F) for every individual subject, was determined by adding up the score for each of the selected (Eysenck) traits (T) for that particular factor, and dividing by the number of selected traits $(\mathrm{N})$ involved (Formula 1):

$$
F=\frac{1}{N} \sum_{n=1}^{N} T_{n}
$$

The three factors thus produced for each subject were then each rounded to the closest 5-point Likert scale (integer) values to create an individual personality vector for each subject (Eysenck \& Eysenck, 1985, p. 192).

\section{A. Results of Personality Questionnaires}

Note, instead of the 27 traits used by Eysenck, only 12 of these were measured in the present study. This means that the combined traits used by us may not fully reflect the original Eysenck factors. To check this, we performed a confirmatory factor analysis in which it was assessed in how far the correlation structure of the measured variables fitted with the original factor model of Eysenck. As suspected, none of the chosen goodness-of-fit indices ((Adjusted) population Gamma $=(0.664), 0.768$, Joreskog GFI $=0.611$, Joreskog AGFI $=$ 0.438 , Bentler-Bonnet Normed Fit Index $=0.224$, BB Non$\mathrm{NFI}=0.083, \mathrm{BB}$ Comparative $\mathrm{FI}=0.252, \mathrm{RMSEA}=0.123$ ) lend support for the model and both the ML- and independence model Chi square were highly significant (resp. 127.27 and 163.91, with degrees of freedom of 54 and 66). We therefore decided not to base our interpretations on Eysenck's model, but to analyse the correlations among the 12 selected variables on their own right. 
TABLE 2

LOADINGS OF FACTORS ABSTRACTED FROM THE 12 MEASURED TRAITS.

\begin{tabular}{|c|c|c|c|c|}
\hline Variable & $\begin{array}{c}\text { Factor } \\
1\end{array}$ & $\begin{array}{c}\text { Factor } \\
2\end{array}$ & $\begin{array}{c}\text { Factor } \\
3\end{array}$ & $\begin{array}{c}\text { Factor } \\
4\end{array}$ \\
\hline Sociability & 0.366230 & -0.728960 & -0.175871 & -0.026398 \\
\hline Shyness & -0.658963 & 0.542248 & 0.016767 & 0.176433 \\
\hline Vulnerability & -0.424035 & -0.559047 & -0.052254 & -0.472986 \\
\hline General Activity Level & 0.677153 & 0.223206 & 0.251580 & 0.118710 \\
\hline Assertiveness & 0.444187 & 0.060199 & -0.604208 & 0.405537 \\
\hline Anxiety & -0.249595 & -0.575984 & -0.086875 & 0.629583 \\
\hline Tension & -0.596330 & 0.023251 & -0.137943 & 0.501070 \\
\hline Creativity & 0.723497 & -0.104735 & 0.348556 & 0.060268 \\
\hline Excitement- Seeking & 0.652433 & 0.271774 & 0.196174 & 0.044795 \\
\hline Dominance & 0.514370 & -0.064347 & -0.699076 & -0.211223 \\
\hline Aggressiveness & 0.143104 & 0.485739 & -0.590123 & -0.175436 \\
\hline Impulsiveness & 0.887287 & -0.065932 & 0.125375 & 0.204800 \\
\hline Expl.Var & 3.843357 & 1.852954 & 1.501525 & 1.204514 \\
\hline Prp.Totl & 0.320280 & 0.154413 & 0.125127 & 0.100376 \\
\hline
\end{tabular}

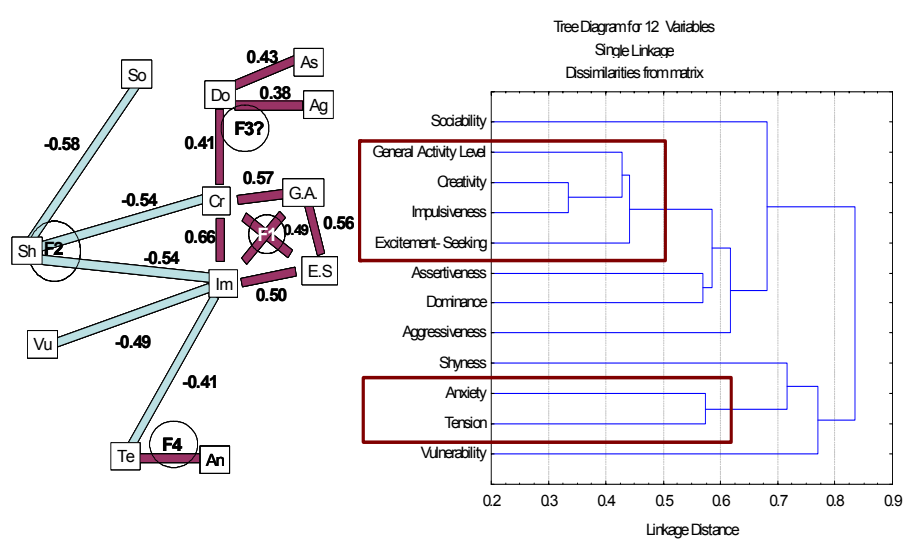

Fig 8. Left: Significant $(\mathrm{p}<0.05)$ Spearman Rank Correlations $\left(\mathrm{r}_{\mathrm{s}}\right)$ among the 12 attributes. Dark bars: positive correlations, light bars: negative correlations. Length of the bars is proportional to the correlation coefficients for $r_{s}>0$ and proportional to 1-rs for $r_{x}<0$. So $=$ Sociality; $\mathrm{Sh}=$ Shyness; $\mathrm{Vu}=$ Vulnerability; G.A. = General Activity Level; As = Assertiveness; $\mathrm{An}=$ Anxiety; $\mathrm{Te}=$ Tension; $\mathrm{Cr}=$ Creativity; E.S. $=$ Excitement Seeking; Do = Dominance; Ag = Aggressiveness; $\mathrm{Im}=$ Impulsiveness. Note the correspondence with the factors F1 - F4 from the factor analysis (Table II). Right: Cluster analysis based on $1-r_{\mathrm{s}}$ as distance metric and Ward's Average as cluster criterion. A cluster analysis on the raw data instead of the correlation coefficients gave largely similar results.

An exploratory factor analysis on the 12 traits shows that $70 \%$ of the variance in the data can be explained by four factors (Table 2). The main traits building up the first factor are Creativity and Impulsiveness. At first sight, this seems to correspond with the "Psychoticism" factor. However, instead of Aggressiveness, General Activity Level and Excitement Seeking also contribute strongly to this factor. We tentatively suggest characterizing this combination as "Proactive" attitudes. This is backed up by the fact that "Shyness" correlates negatively with this factor. Factor 2 appears to reflect the degree of what might be called "Social reluctance": Shyness contributes relatively strongly to it and there is a strong negative correlation with Sociability. Factor 3 seems to characterize "Timidity", as Assertiveness, Dominance and Aggressiveness are all associated negatively with it.
"Nervousness" typifies factor 4: both Anxiety and Tension load rather high on it.

The factor analysis is backed up by a Principle Component Analysis (PCA; the principle components are almost identical to the factors), which is based on less assumptions and a non-parametric approach (clustering on Spearman Rank correlations; Fig. 8).

When testing for correlations between approach distances and personality data, "Proactive" is the only factor that correlates with social distance, in the sense that subjects that score higher on this factor come less close to the robot $\left(\mathrm{r}_{\mathrm{s}}=\right.$ $0.647, \mathrm{p}<0.05)$. Also the effects of gender, age and technical or robotics experience were investigated. Although males appear to score higher on the second ("Social reluctance") factor (Mann Whitney $U$ Test, $U=48, z=2.389, p<0.002$ ), none of these other demographic factors associated significantly with social distance.

\section{CONCLUSIONS}

We have found that a majority of human subjects $(60 \%)$ when approaching a robot, or when being approached by a robot, prefer approach distances that are compatible with those expected for normal social interactions between humans. This partially confirms our original hypothesis in that it seems that humans respect human-robot approach distances in a way which is comparable to human-human social distances. However, in our experiments a large minority of subjects (40\%) took up an initial approach distance to the robot, which was so close that it would be perceived as either threatening (if involving strangers) or intimate (in the case of close friends) if observed between two humans. These subjects clearly did not perceive the robot in a way that was comparable with normal human-human social distances, which might imply that these subjects did not perceive the robot as a 'social entity' with respect to distances in the same way as another human being.

We studied subjects' personalities to see if there were common factors, which could be used to predict the likely approach distance preferred by the subjects. Factor analysis resulted in four factors that we tentatively label "Proactiveness", "Social Reluctance", "Timidity" and "Nervousness". Correlations with the social distance experiments show a positive correlation for "Proactive", i.e. the more proactive a person judged him/herself the longer the human-to-robot approach distances measured.

At this stage our characterization of these four factors, as an alternative to Eysenck's factors, is preliminary. For this particular study, we do not suggest to use them as a universal scale for human robot interactions. Potentially, factors might be identified as being most suitable for human-robot interaction studies; possibly specific to particular contexts, task environments, particular robots, and/or experimental settings, but this requires deeper analysis and confirmation in future studies (for an example of such a scale, see [27]). Moreover, the sample of subjects we used was self-selected (University staff/students). A subject sample that is more representative of 'potential users' of a robot companion might yield different results and also cultural differences might have to be taken into account [28]). Also, in future work we need to 
consider that the markings on the floor could influence subjects' judgements. Furthermore, social distances may be affected by the robots' appearance, subjects' own identification of whether they are seen as social entities, tasks of robots and so on. These factors may obviously play a role in human - robot interactions and are important topics for further studies. However, if we succeed in identifying and confirming a set of such factors, then based on a person's personality assessment, one could adjust human-robot distances according to the subject's personality profile. This would provide an important step towards personalized robot companions [26].

\section{REFERENCES}

[1]. T. Fong, I. Nourbakhsh, and K. Dautenhahn, (2003). A survey of socially interactive robots, Robotics and Autonomous Systems, vol. 42, pp. 143-166.

[2]. S. Thrun, (1998). When robots meet people, IEEE (see also IEEE Expert) Intelligent Systems, vol. 13, pp. 27 - 29.

[3]. J. Goetz and S. Kiesler, (2002). Cooperation with a Robotic Assistant. Proc. CHI'02 Conference on Human Factors in Computing Systems, New York, USA, 2002.

[4]. M. Scopelliti, M. V. Giuliani, A. M. D'Amico, and F. Fornara, (2004). "If I had a robot at home.... Peoples' representation of domestic robots", in Designing a more inclusive world. S. Keates, J. Clarkson, P. Langdon, and P. Robinson, Eds., Springer, pp. 257-266.

[5]. K. Severinson-Eklundh, A. Green, H. Hüttenrauch, (2003). Social and Collaborative Aspects of Interaction with a Service Robot. Robotics and Autonomous Systems 42 (2003) pp. 223-234.

[6]. Y. Nakauchi and R. Simmons, (2002). A Social Robot that Stands in Line, Autonomous Robots, Vol. 12, No. 3, pp. 313 -324.

[7]. C. L. Breazeal, (2002). Designing sociable robots. Massachusetts: The MIT Press, 2002.

[8]. B. Friedman, P. H. Kahn (Jr.), and J. Hagman, (2003). Hardware companions? What online AIBO discussion forums reveal about the human-robotic relationship, Digital Sociability, vol. 5, pp. 273-280.

[9]. T. Kanda, H. Ishiguro, M. Imai, and T. Ono, Body Movement Analysis of Human-Robot Interaction, International Joint Conference on Artificial Intelligence (IJCAI 2003), pp.177-182.

[10]. H. G. Okuno, K. Nakadai, and H. Kitano (2002). "Realizing AudioVisually Triggered ELIZA-Like Non-verbal Behaviors," PRICAI2002, LNAI 2417, Springer-Verlag, pp.552-562.

[11]. S. Woods, K. Dautenhahn, J. Schulz, (2004). The Design Space of Robots: Investigating Children's Views. Proc. IEEE Ro-man 2004, IEEE Press, pp. 47-52.

[12]. P Hinds, T Roberts, H Jones, (2004). Whose Job Is It Anyway? A Study of Human-Robot Interaction in a Collaborative Task. Human-Computer Interaction, Vol. 19 pp151-181.

[13]. Z. Khan, (1998). Attitude towards intelligent service robots, NADA KTH Technical Report, Stockholm 1998.

[14]. K. Dautenhahn, (2002). Design Spaces and Niche Spaces of Believable Social Robots. Proc. IEEE Ro-man 2002, IEEE Press, pp. 192-197.

[15]. T Minato, M Shimada, H Ishiguro and S Itakura, (2004). Development of an Android Robot for Studying Human-Robot Interaction. Proc. IEA/AIE Conf. 2004, pp. 424-334

[16]. K. Dautenhahn, K. L. Koay, R. te Boekhorst, M. Walters, (2005). Perception of Robot 'Personalities' Based on Robot's Behaviour Styles. Submitted for publication.

[17]. E.T. Hall, (1966). The Hidden Dimension: Man's Use of Space in Public and Private. The Bodley Head Ltd, London, UK.

[18]. E. T. Hall, (1968). Proxemics. Current Anthropology 9(2-3): 83-108. 1968

[19]. D Lambert, (2004). Body Language. HarperCollins.

[20]. M Walters, K Dautenhahn, K. L. Koay, R. te Boekhorst, C. Nehaniv, I. Werry, D. Lee, (2005). Close Encounters: Spatial distances between People and a Robot of Mechanistic Appearance. Submitted for publication.

[21]. H. I. Christensen, E Pacchierotti, (2005). Embodied social interaction for robots. Proc. AISB'05 Symposium on Robot Companions: Hard Problems and Open Challenges in Human-Robot Interaction, pp.40-45.
[22]. B. Robins, K. Dautenhahn, J. Dubowski (2004). Investigating Autistic Children's Attitudes Towards Strangers with the Theatrical Robot - A New Experimental Paradigm in Human-Robot Interaction Studies. Proc. IEEE Ro-man 2004, IEEE Press, pp. 557-562.

[23]. A. Green, H. Hüttenrauch, K Severinson Eklundh (2004). Applying the Wizard of $\mathrm{Oz}$ Framework to Cooperative Service discovery and Configuration. Proc. IEEE Ro-man 2004, IEEE Press, pp. 575-580.

[24]. H. J. Eysenck, (1991). Dimensions of personality: 16, 5, or 3?-Criteria for a taxonomic paradigm, Personality and Individual Differences, 12, 773-790.

[25]. H. J. Eysenck and M. W. Eysenck, (1985). Personality and individual differences: A natural science approach. New York: Plenum, 1985.

[26]. K. Dautenhahn, (2004). Robots We Like to Live With?! - A Developmental Perspective on a Personalized, Life-Long Robot Companion, Proc. IEEE Ro-man 2004, IEEE Press, pp. 17-22.

[27]. T. Nomura, T. Kanda, T. Suzuki and K. Kato (2004). Psychology in Human-Robot Communication: an attempt through investigation of negative attitudes and anxiety towards robots. In Proc. $13^{\text {th }}$ IEEE International Workshop on Robot and Human Interactive Communication, pp. 35-40

[28]. C. Bartneck, T. Nomura, T. Kanda, T. Suzuki and K. Kato (2005). A cross-cultural study on attitudes towards robots. Proc. $11^{\text {th }}$ International Conference on Human-Computer Interaction (to appear). 